\title{
USO DA PROGRAMAÇÃO LINEAR INTEIRA NA DESIGNAÇÃO DE ENCARGOS DIDÁTICOS: UM ESTUDO DE CASO
}

\section{USE OF THE INTEGER LINEAR PROGRAMMING IN THE DESIGNATION OF DIDACTICAL ASSIGNMENTS: A CASE STUDY}

\author{
Ana Paula Moreira ${ }^{1}$ \\ Daniele Silva ${ }^{2}$ \\ José Carlos de Moraes Filho ${ }^{3}$
}

Resumo: A proposta deste trabalho é a apresentação de um modelo de programação linear inteira para o problema de designação de encargos didáticos em uma instituição de ensino superior que maximize as preferências e habilidades dos professores. Para validação e avaliação da eficácia do modelo foi realizado um estudo de caso preliminar no Departamento de Matemática da Universidade Tecnológica Federal do Paraná Campus Cornélio Procópio. Neste contexto, os resultados obtidos são promissores, com melhoras em índices relacionados à satisfação, habilidade e carga horária docente em comparação com a designação manual adotada pelo departamento e também com outra formulação para o problema apresentada na literatura.

Palavras-chave: Modelagem matemática. Programação linear inteira. Designação de encargos didáticos.

Abstract: This paper aim is to present an integer linear programming model to the problem of the designation of didactical assignments in a university that maximizes professor's abilities and preferences. To validate and evaluate the effectiveness of the model, a preliminary case study was carried out at the Department of Mathematics of the Federal Technological University of Paraná - Cornélio Procópio Campus. In this context, the results obtained are promising, with improvements in indexes related to satisfaction, skill and teacher workload compared to the manual designation adopted by the department and also with another formulation for the problem presented in the literature.

Key words: Math modeling. Integer linear programming. Designation of didactical assignments.

Data de submissão: 14.10 .2020

Data de aprovação: 08.02.2021

Identificação e disponibilidade:

(https://revista.univap.br/index.php/revistaunivap/article/view/2557, http://dx.doi.org/10.18066/revistaunivap.v27i56.2557).

\footnotetext{
1 Mestranda no Programa de Pós-Graduação em Ensino de Ciências e Educação Matemática na Universidade Estadual de Londrina (UEL), E-mail: ana moreira@live.com.

2 Professora adjunta na Universidade Tecnológica Federal do Paraná - Campus Cornélio Procópio, Email:danielesilva@utfpr.edu.br.

3 Pós-graduando no Programa de Pós-Graduação em Gestão de Projetos pelo Serviço Nacional de Aprendizagem Industrial (SENAI), E-mail: josecarlosdemoraesfilho@gmail.com.
} 


\section{INTRODUÇÃO}

A designação de encargos didáticos é uma prática comum e necessária em toda instituição de ensino antes do início de cada período letivo. Apesar de ser uma atividade bem definida, sua alta complexidade computacional (EVEN; ITAI; SHAMIR, 1975) e as diversas nuances de acordo com as particularidades de cada instituição, desperta desde a década de 60 (GOTLIEB, 1963; CSIMA; GOTLIEB, 1964; WELSH; POWELL, 1967) o interesse de diversos pesquisadores na busca de modelos e técnicas de resolução mais representativos e eficientes.

Nesse sentido, diversos métodos e algoritmos podem ser aplicados na modelagem e resolução deste problema, tais como: Algoritmos Exatos, Heurísticas e Metaheurísticas - Simulated Annealing, Algoritmos Genéticos, Busca Tabu, GRASP (Procedimento de Busca Gulosa Adaptativa Aleatória), Colônia de Abelhas, e Abordagens Híbridas, entre outros (PILLAY, 2013).

Neste trabalho, o enfoque são algoritmos exatos, mais especificamente algoritmos e modelos de Programação Linear e suas variações. A Programação Linear é uma subárea da Pesquisa Operacional que consiste na modelagem e resolução de problemas que envolvem a minimização (ou maximização) de uma função linear, sujeita a restrições também lineares. Quando as variáveis desses problemas devem assumir valores inteiros estes são denominados problemas de Programação Linear Inteira e se algumas variáveis devem ser inteiras e as demais não tem essa obrigatoriedade tem-se problemas de Programação Linear Inteira Mista.

Nesse contexto, os primeiros trabalhos foram desenvolvidos por Lawrie (1969) e Akkoyunlu (1973) os quais apresentaram modelos de Programação Linear Inteira para algumas versões do problema e obtiveram bons resultados computacionais para a época. No cenário de uma universidade, o trabalho desenvolvido em Breslaw (1976) foi um dos pioneiros. Neste é apresentada uma solução para o problema utilizando Programação Linear.

Com os progressos na capacidade de resolução e avanços tecnológicos se tornou possível a modelagem por meio da Programação Linear e Programação Linear Inteira com considerações práticas, o que torna os modelos mais representativos, como os apresentados em Birbas, Daskalaki e Housos (1997) e Avella e Vasil'Ev (2005). Mais recentemente, tem-se os trabalhos de Ferreira et al. (2011) que usam Programação Linear Inteira com o objetivo de maximizar a satisfação dos professores; Heitmann e Brüggemann (2013) que formularam um modelo de Programação Linear Inteira Mista, no qual os estudantes podem especificar individualmente suas preferências para classes e grupos, obtendo resultados satisfatórios; Domenech e Lusa (2015) que também utilizaram Programação Linear Inteira Mista para designação de aulas a professores universitários com bons resultados; Abdellahi e Eledum (2017) que utilizaram Programação Linear Inteira com o intuito de minimizar o tempo de espera entre as aulas para alunos e professores, dentre diversos outros trabalhos, o que mostra que o tema é atual e relevante.

No presente trabalho, o problema de designação de encargos didáticos é abordado tomando como estudo de caso a distribuição de disciplinas de graduação à professores do Departamento Acadêmico de Matemática (DAMAT) da Universidade Tecnológica Federal do Paraná (UTFPR), Campus Cornélio Procópio, no primeiro semestre letivo de 2017. É proposto um modelo de Programação Linear Inteira que visa a maximização da satisfação docente e atribuição de disciplinas aos mesmos considerando a habilidade em ministrá-las. É válido ressaltar que é possível adaptar o modelo para o uso em outras instituições de ensino. 
A organização deste trabalho se faz na seguinte ordem, na Seção 2 é apresentado o modelo de Programação Linear Inteira proposto; na Seção 3 é apresentada a contextualização do estudo de caso e o processo da coleta de dados necessária para a realização do estudo; a análise dos resultados é realizada na Seção 4; e as considerações finais são apresentadas na Seção 5.

\section{MODELO MATEMÁTICO}

De acordo com Carvalho (2011) três aspectos básicos devem ser observados ao formular e solucionar o problema de designação de encargos didáticos, são eles: aspectos organizacionais, aspectos pedagógicos e aspectos pessoais. Os aspectos organizacionais são relativos à instituição de ensino, referem-se às normas vigentes da instituição que devem ser respeitadas. Os aspectos pedagógicos referem-se ao desenvolvimento de um ensino de qualidade. E por fim, os aspectos pessoais são relacionados as preferências e/ou necessidades do corpo docente e estudantes.

Levando em conta estes três aspectos, o modelo matemático desenvolvido neste trabalho, o qual tem como base a formulação básica apresentada em Ferreira et al. (2011), considera que $\boldsymbol{T}$ turmas devem ser divididas para $\boldsymbol{P}$ professores em $\boldsymbol{H}$ horários ao longo de $\boldsymbol{D}$ dias semanais. Para a atribuição professor turma utiliza-se a variável de decisão binária $x(p, t)$, tal que, para todo $p=1, \ldots, P$ e $t=1, \ldots, T$, tem-se: $x(p, t)=1$ se o professor $p$ assume a turma $t$ e $x(p, t)=0$ caso contrário.

Os horários de cada turma são fixados e armazenados da seguinte forma: $\boldsymbol{H T}(\boldsymbol{t}, \boldsymbol{h}, \boldsymbol{d})=\mathbf{1}$ se a turma $\boldsymbol{t}$ tem aula no horário $\boldsymbol{h}$ no dia $\boldsymbol{d}$ e $\boldsymbol{H T}(\boldsymbol{t}, \boldsymbol{h}, \boldsymbol{d})=\mathbf{0}$ caso contrário; para todo $t=1, \ldots, T, h=1, \ldots, H, d=1, \ldots, D$.

De forma similar, a disponibilidade do professor $\boldsymbol{p}$ em assumir a turma $\boldsymbol{t}$ no horário $\boldsymbol{h}$ e dia $\boldsymbol{d}$ é armazenada como: $\boldsymbol{D}(\boldsymbol{p}, \boldsymbol{h}, \boldsymbol{d})=\mathbf{1}$ se o professor $\boldsymbol{p}$ tem disponibilidade no horário $\boldsymbol{h}$ e dia $\boldsymbol{d}$ e $\boldsymbol{D}(\boldsymbol{p}, \boldsymbol{h}, \boldsymbol{d})=\mathbf{0}$ caso contrário; para todo $\boldsymbol{p}=$ $1, \ldots, P, h=1, \ldots, H, d=1, \ldots, D$.

A carga horária atribuída a cada docente deve estar compreendida entre um valor mínimo $\boldsymbol{H}_{\min }(\boldsymbol{p})$ e um valor máximo $\boldsymbol{H}_{\max }(\boldsymbol{p})$ os quais são definidos pela instituição.

Além destes fatores, considera-se a preferência do professor $\boldsymbol{p}$ em assumir uma turma $\boldsymbol{t}$, a qual é dada por um peso $\boldsymbol{e}(\boldsymbol{p}, \boldsymbol{t})$, que assume valores inteiros que variam de 0 a 5 , onde 0 indica nenhum interesse e 5 maior preferência; e também a habilidade do professor $\boldsymbol{p}$ em ministrar a disciplina relacionada à turma $\boldsymbol{t}$, que é avaliada pelo peso $\boldsymbol{a}(\boldsymbol{p}, \boldsymbol{t})$, que é um número inteiro o qual varia de 0 a 2 , onde 0 é a circunstância em que o professor não possui habilidade em ministrar a disciplina e 2 que indica maior habilidade em lecionar a disciplina. O objetivo do modelo é maximizar a habilidade e satisfação total dos professores em relação às disciplinas, os quais são dados por:

$$
\sum_{p=1}^{P} \sum_{t=1}^{T}((1-\lambda) e(p, t)+\lambda a(p, t)) x(p, t)
$$

Onde $\mathbf{0} \leq \lambda \leq \mathbf{1}$ é um parâmetro que determina se será dada uma maior prioridade à habilidade ou à satisfação. Note que quanto maior o valor atribuído a $\lambda$ o modelo priorizará a habilidade do professor e quanto menor a satisfação. 
Assim, temos a seguinte formulação:

Maximizar $\sum_{p=1}^{P} \sum_{t=1}^{T}((1-\lambda) e(p, t)+\lambda a(p, t)) x(p, t)$

Sujeito a:

$$
\sum_{t=1}^{T} H T(t, h, d) x(p, t) \leq 1, \text { para todo } t, h, d
$$

$\sum_{p=1}^{P} x(p, t)=1$, para todo $t$

$\sum_{t=1}^{T} \sum_{h=1}^{H} \sum_{d=1}^{D} H T(t, h, d) x(p, t) \geq H_{\min }(p)$, para todo $p$

$\sum_{t=1}^{T} \sum_{h=1}^{H} \sum_{d=1}^{D} H T(t, h, d) x(p, t) \leq H_{\max }(p)$, para todo $p$

$(D(p, h, d)-H T(t, h, d)) x(p, t) \geq 0$, para todo $p, t, d, h$

$x(p, t)=0$ ou 1, para todo $p, t$.

As restrições (3) e (4) são básicas para a elaboração de um quadro de horários, a primeira exige que o professor não possa assumir mais de uma turma em um mesmo horário e dia; e a segunda garante que nenhuma turma ficará sem professor. Já as restrições (5) e (6) garantem que a carga horária semanal de cada professor estará compreendida entre valores mínimo e máximo pré-estabelecidos. O conjunto de restrições (7) impede que um professor assuma uma turma em um horário em que o mesmo não possua disponibilidade. Por fim, a restrição (8) define as variáveis como binárias.

\section{ESTUDO DE CASO}

Com intuito de avaliar a eficácia do modelo apresentado na Seção 2, este foi testado utilizando dados da distribuição de disciplinas do primeiro semestre letivo de 2017 do Departamento Acadêmico de Matemática da Universidade Tecnológica Federal do Paraná, Campus Cornélio Procópio (DAMAT). Neste semestre foram distribuídas manualmente por meio de uma comissão, 90 turmas de graduação a 32 professores em 15 horários em cada um dos 5 dias semanais.

Para tanto, foi necessário realizar um levantamento acerca da preferência e da disponibilidade de horário de cada professor e também uma avaliação de sua habilidade em ministrar uma determinada disciplina.

Os dados referentes à preferência e disponibilidade foram coletados por meio de um formulário eletrônico, no qual cada docente se identificava, informava sua disponibilidade de horário, se atua em programa de pós-graduação e/ou possui cargo administrativo, visto que pelo regulamento da instituição (UTFPR, 2007) estes fatores interferem na carga horária do docente; e atribuía um peso de 0 a 5 às turmas indicando sua preferência conforme explanado na Seção 2.

É válido ressaltar que neste trabalho considera-se turma a oferta de uma determinada disciplina a um determinado curso de graduação do campus. Por exemplo, Cálculo Numérico para Engenharia Elétrica é uma turma, Cálculo Numérico para Licenciatura em Matemática é outra turma. O período do curso, dias da semana e horários em que esta oferta é realizada são determinados e fixados previamente pelas coordenações dos respectivos cursos. E com o porte destas informações, os 
professores manifestaram sua preferência.

Para a avaliação da habilidade foi feita uma análise curricular com base no Currículo Lattes de cada docente. Nesse sentido, atribuiu-se pesos de 0 a 2 às turmas seguindo os seguintes critérios:

Peso 0: se a disciplina relacionada à turma não pertence a área de atuação docente e o mesmo não possui experiência curricular relacionada à disciplina;

Peso 1: se a disciplina relacionada à turma não pertence a área de atuação docente, no entanto o mesmo possui experiência curricular com a disciplina;

Peso 2: se a disciplina relacionada à turma pertence a área de atuação do docente.

Com isso, foram realizados experimentos computacionais com variações de um décimo no valor do parâmetro $\lambda$. Os mesmos foram executados em uma plataforma Intel Core i3, $2.30 \mathrm{GHz}$ com 4,00 GB de memória RAM em um sistema operacional Windows de 32 Bits, com apoio do GLPK (GNU Linear Programming Kit) 4 pacote desenvolvido para a resolução de problemas de programação linear e programação inteira mista de grande porte e outros problemas relacionados.

\section{ANÁLISE DE RESULTADOS}

A presente análise considera a comparação entre a designação manual de encargos didáticos realizada no primeiro semestre letivo de 2017 frente ao modelo proposto neste trabalho.

Para isso, foram considerados três fatores: habilidade, satisfação e carga horária. As características e relevâncias de cada um destes fatores são descritas a seguir.

\subsection{HABILIDADE}

Um fator importante a ser considerado na designação de disciplinas é a habilidade do professor em ministrá-las. Ou seja, atribuir disciplinas com as quais o docente possua experiência curricular e/ou que estejam relacionadas com sua área de formação e atuação profissional. Devido a esta relevância a habilidade é um dos parâmetros da análise dos resultados obtidos por meio dos testes computacionais. Para tal, considerou-se a Habilidade Global, isto é, a soma de todos os pesos $\boldsymbol{a}(\boldsymbol{p}, \boldsymbol{t})$ atribuídos aos professores; a percentagem de aumento da habilidade global em relação a designação manual, o Índice Médio de Habilidade e a Análise de Variância (VIEIRA, 2006) dos mesmos.

O Índice Médio de Habilidade $(\boldsymbol{I H})$ é calculado da seguinte forma: considera-se que ao professor $\boldsymbol{p}$ foram atribuídas $\boldsymbol{q}(\boldsymbol{p})$ turmas não obrigatoriamente as de sua maior habilidade, também, considere que as $\boldsymbol{q}(\boldsymbol{p})$ turmas de sua maior habilidade sejam $t_{1}, t_{2}, \ldots, t_{q(p)}$. O índice de habilidade individual $I(h)$ é dado pela razão entre a soma dos pesos das $\boldsymbol{q}(\boldsymbol{p})$ turmas designadas ao professor $\boldsymbol{p}$, e o peso máximo se as $\boldsymbol{q}(\boldsymbol{p})$ turmas designadas a ele fossem as de sua maior habilidade, ou seja $\boldsymbol{I}(\boldsymbol{h})=$

4 MAKHORIN, Andrew. GLPK (GNU Linear Programming Kit). Moscow, Russia: Department for Applied Informatics, Moscow Aviation Institute, c2012. Disponível em: www.gnu.org/software/glpk. Acesso em: 13 ago. 2021. 
$\frac{\sum_{t=1}^{T} a(p, t) x(p, t)}{\sum_{j=1}^{q(P)} \sum_{t=1}^{T} a\left(p, t_{j}\right)}$

A média dos índices $\boldsymbol{I}(\boldsymbol{h})$ resulta no Índice Médio de Habilidade dos professores, $\boldsymbol{I} \boldsymbol{H}=\frac{\mathbf{1}}{\boldsymbol{P}} \sum_{\boldsymbol{p}=\mathbf{1}}^{\boldsymbol{I}} \boldsymbol{I}(\boldsymbol{h})$. A Habilidade Global, a percentagem de aumento e o Índice Médio de Habilidade são apresentados na Tabela 1.

Para a Análise de Variância foi considerado como grupo a forma de designação, ou seja, se a mesma foi manual ou utilizando o modelo proposto com as variações no valor do parâmetro $\lambda$. Desse modo, foi necessário o cálculo dos Graus de Liberdade $(\boldsymbol{G} \boldsymbol{L})$ entre os grupos $\left(\boldsymbol{G} \boldsymbol{L}_{\boldsymbol{G}}\right)$, que é o número de grupos menos um, e dentro dos grupos $\left(\boldsymbol{G} \boldsymbol{L}_{\boldsymbol{R}}\right)$ que é o total de dados menos o número de grupos; da Soma de Quadrados $(\boldsymbol{S} \boldsymbol{Q})$ entre os grupos $\left(\boldsymbol{S} \boldsymbol{Q}_{G}\right)$ e dentro dos grupos $\left(\boldsymbol{S} \boldsymbol{Q}_{R}\right)$, obtendo a medida da variabilidade dos dados em ambos os casos; do Quadrado Médio $(\boldsymbol{Q} \boldsymbol{M})$ entre os grupos $\left(\boldsymbol{Q} \boldsymbol{M}_{\boldsymbol{G}}=\frac{\boldsymbol{S} \boldsymbol{Q}_{G}}{\boldsymbol{G} \boldsymbol{L}_{G}}\right)$ e dentro dos grupos $\left(\boldsymbol{Q} \boldsymbol{M}_{\boldsymbol{R}}=\frac{\boldsymbol{S} \boldsymbol{Q}_{\boldsymbol{R}}}{\boldsymbol{G} \boldsymbol{L}_{\boldsymbol{R}}}\right)$; e da estatística $\boldsymbol{F}=\frac{\boldsymbol{Q} \boldsymbol{M}_{G}}{\boldsymbol{Q} \boldsymbol{M}_{\boldsymbol{R}}}$, a qual é utilizada para verificar se há ou não uma diferença significativa nos índices de habilidade entre os grupos. Estes dados são apresentados na Tabela 2.

A Soma de Quadrados entre os grupos $\left(\boldsymbol{S} \boldsymbol{Q}_{G}\right)$ é dada por $\boldsymbol{S} \boldsymbol{Q}_{\boldsymbol{G}}=$ $\sum_{G=1}^{11} \boldsymbol{n}_{G}\left(\boldsymbol{I} \boldsymbol{H}_{\boldsymbol{G}}-\boldsymbol{I} \boldsymbol{H}_{M}\right)^{\mathbf{2}}$, onde $\boldsymbol{G}$ é o número de grupos, $\left(\boldsymbol{n}_{G}\right)$ o número de elementos do grupo $\boldsymbol{G}, \boldsymbol{I H}_{\boldsymbol{G}}$ o Índice Médio de Habilidade do grupo $\boldsymbol{G}$ e $\boldsymbol{I} \boldsymbol{H}_{\boldsymbol{M}}$ a média dos $\boldsymbol{I} \boldsymbol{H}_{\boldsymbol{G}}$. E a Soma de Quadrados dentro dos grupos $\left(S Q_{R}\right)$ é dada por $S Q_{R}=\sum_{G=1}^{11} n_{G}\left(I(h)_{G k}-\right.$ $\left.\boldsymbol{I} \boldsymbol{H}_{\boldsymbol{G}}\right)^{2}$, onde $\boldsymbol{I}(\boldsymbol{h})_{\boldsymbol{G} \boldsymbol{k}}$ é o Índice de Habilidade Individual $\boldsymbol{k}$ do grupo $\boldsymbol{G}$.

Tabela 1 - Tabela de Habilidade.

\begin{tabular}{|c|c|c|c|c|}
\hline Grupo & Modelo & $\begin{array}{l}\text { Habilidade } \\
\text { Global }\end{array}$ & $\begin{array}{c}\text { Aumento } \\
\text { da } \\
\text { Habilidade } \\
\text { Global (\%) }\end{array}$ & $\begin{array}{l}\text { Índice } \\
\text { Médio } \\
\text { (IH) }\end{array}$ \\
\hline 1 & $\begin{array}{c}1^{\circ} \text { Semestre } \\
\text { de } 2017\end{array}$ & 173 & 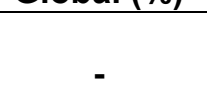 & 0,9766 \\
\hline 2 & $\square=0$ & 151 & $-12,72$ & 0,8451 \\
\hline 3 & $\square=\mathbf{0 , 1}$ & 177 & 2,31 & 0,9948 \\
\hline 4 & $\square=\mathbf{0 , 2}$ & 177 & 2,31 & 0,9948 \\
\hline 5 & $\square=\mathbf{0 , 3}$ & 177 & 2,31 & 0,9948 \\
\hline 6 & $\square=\mathbf{0 , 4}$ & 177 & 2,31 & 0,9948 \\
\hline 7 & $\square=\mathbf{0 , 5}$ & 177 & 2,31 & 0,9948 \\
\hline 8 & $\square=\mathbf{0 , 6}$ & 177 & 2,31 & 0,9948 \\
\hline 9 & $\square=\mathbf{0 , 7}$ & 177 & 2,31 & 0,9948 \\
\hline 10 & $\square=\mathbf{0 , 8}$ & 178 & 2,89 & 1,000 \\
\hline 11 & $\square=\mathbf{0 , 9}$ & 178 & 2,89 & 1,000 \\
\hline
\end{tabular}

Fonte: Elaborado pelos autores. 
Tabela 2 - Quadro de Análise de Variância - Habilidade.

\begin{tabular}{cccccc}
\hline $\begin{array}{c}\text { Origem de } \\
\text { Variações }\end{array}$ & $\begin{array}{c}\text { Grau de } \\
\text { Liberdade } \\
\text { (GL) }\end{array}$ & $\begin{array}{c}\text { Soma de } \\
\text { Quadrados } \\
\text { (SQ) }\end{array}$ & $\begin{array}{c}\text { Quadrado } \\
\text { Médio } \\
\text { (QM) }\end{array}$ & F & $\begin{array}{c}\text { F - crítico } \\
\square=0,05\end{array}$ \\
\hline $\begin{array}{c}\text { Entre } \\
\text { Grupos } \\
\text { Dentro } \\
\text { dos } \\
\text { Grupos }\end{array}$ & 10 & 0,6577 & 0,0658 & 9,1429 & 1,8585 \\
Total & 341 & 2,4529 & 0,0072 & & \\
\hline
\end{tabular}

Fonte: Elaborado pelos autores.

Por meio da Tabela 1 é possível observar que em todos os testes, exceto para $\lambda=\mathbf{0}$ (formulação apresentada em Ferreira et al. (2011)) em que houve redução de $12,72 \%$ da habilidade global em relação à designação manual e de $15 \%$ em relação aos demais testes, o modelo proposto apresenta melhoras neste índice, um aumento de em média $2,44 \%$, e consequentemente, no índice médio de habilidade. É válido ressaltar que para $\lambda=\mathbf{0 , 8}$ e $\boldsymbol{\lambda}=\mathbf{0 , 9}$ o índice médio é igual a 1, ou seja, as disciplinas relacionadas às turmas designadas a todos os professores foram as de sua maior habilidade.

Analisando a Tabela 2, nota-se que a estatística $\boldsymbol{F}$ é superior ao valor crítico para um nível de significância $\alpha=0,05$, ou seja, uma confiabilidade de $95 \%$. O que indica que há um grupo ou mais com índices de habilidade significativamente distintos.

Para identificação desses grupos foi realizado o Teste da Diferença Mínima Significativa - Least Significant Difference (LSD) Test (VIEIRA, 2006), o qual consiste em comparar todos os pares de média, no caso todos os pares $\boldsymbol{I H}$, e caso o valor absoluto da diferença entre o par de médias for superior a diferença mínima significativa dada por $\boldsymbol{L S D}=\boldsymbol{t}\left(\frac{\alpha}{2}, \boldsymbol{G} \boldsymbol{L}_{R}\right) \sqrt{2 \frac{S Q_{R}}{n}}$ (onde $\boldsymbol{t}$ é o inverso bicaudal da distribuição $t$ de Student, e $\boldsymbol{n}$ é o número total de dados), estas são significativamente diferentes. O resultado obtido para um nível de significância $\alpha=0,05$, foi que os grupos relacionados à designação manual (Grupo 1) e a $\boldsymbol{\lambda}=\mathbf{0}$ (Grupo 2), diferem significativamente entre si e dos demais grupos. O que confirma que realizar a distribuição de tarefas didáticas de forma manual ou pelo modelo básico apresentado em Ferreira et al. (2011), para este estudo de caso, interferiu negativamente nos índices de habilidade.

\subsection{SATISFAÇÃO}

Além da habilidade em ministrar determinada disciplina é essencial que o professor tenha interesse em lecioná-la. Nesse sentido, a satisfação docente também é um dos fatores da análise de resultados.

De forma similar à habilidade, considerou-se a Satisfação Global, isto é, a soma de todos os pesos $\boldsymbol{e}(\boldsymbol{p}, \boldsymbol{t})$ atribuídos aos professores, a percentagem de aumento da satisfação global em relação a designação manual, o Índice Médio de Satisfação e a Análise de Variância. 
Para o cálculo do Índice Médio de Satisfação (IS) considera-se a atribuição de $\boldsymbol{q}(\boldsymbol{p})$ turmas ao professor $\boldsymbol{p}$, não necessariamente as de sua maior preferência, e que as $\boldsymbol{q}(\boldsymbol{p})$ turmas de sua maior preferência são $\boldsymbol{t}_{1}, \boldsymbol{t}_{2}, \ldots, \boldsymbol{t}_{\boldsymbol{q}(\boldsymbol{p})}$. Assim, tem-se o índice de satisfação individual $I(p)=\frac{\sum_{t=1}^{T} e(p, t) x(p, t)}{\sum_{j=1}^{q(P)} \sum_{t=1}^{T} e\left(p, t_{j}\right)}$, e o Índice Médio de Satisfação $I S=$ $\frac{1}{P} \sum_{p=1}^{P} I(p)$. Estes dados são apresentados na Tabela 3.

Para a Análise de Variância novamente utilizou-se os valores dos Graus de Liberdade $\boldsymbol{G} \boldsymbol{L}_{\boldsymbol{G}}$ e $\boldsymbol{G} \boldsymbol{L}_{\boldsymbol{R}}$, da Soma de Quadrados $\boldsymbol{S} \boldsymbol{Q}_{G}$ e $\boldsymbol{S} \boldsymbol{Q}_{\boldsymbol{R}}$, dos Quadrado Médios $\boldsymbol{Q} \boldsymbol{M}_{\boldsymbol{G}}$ e $\boldsymbol{Q} \boldsymbol{M}_{\boldsymbol{R}}$, e da estatística $\boldsymbol{F}$. Com a diferença que a $\boldsymbol{S} \boldsymbol{Q}_{G}$ é dada por $\boldsymbol{S} \boldsymbol{Q}_{G}=$ $\sum_{G=1}^{11} n_{G}\left(I S_{G}-I S_{M}\right)^{2}$, onde $I S_{G}$ é o Índice Médio de Satisfação do grupo $G$ e $I S_{M}$ a média dos $I S_{G}$; e a $S Q_{R}$ dada por $S Q_{R}=\sum_{G=1}^{11} n_{G}\left(I(p)_{G k}-I S_{G}\right)^{2}$, onde $I(p)_{G k}$ é o Índice de Satisfação Individual $\boldsymbol{k}$ do grupo $\boldsymbol{G}$. Estes dados são exibidos na Tabela 4.

Tabela 3 - Tabela de Satisfação.

\begin{tabular}{|c|c|c|c|c|}
\hline Grupo & Modelo & $\begin{array}{l}\text { Satisfação } \\
\text { Global }\end{array}$ & $\begin{array}{c}\text { Aumento } \\
\text { da } \\
\text { Satisfação } \\
\text { Global (\%) }\end{array}$ & $\begin{array}{l}\text { Índice } \\
\text { Médio } \\
\text { (IS) }\end{array}$ \\
\hline 1 & $\begin{array}{c}1^{\circ} \text { Semestre } \\
\text { de } 2017\end{array}$ & 421 & - & 0,9432 \\
\hline 2 & $\square=\mathbf{0}$ & 434 & 3,09 & 0,9729 \\
\hline 3 & $\square=\mathbf{0 , 1}$ & 429 & 1,90 & 0,9625 \\
\hline 4 & $\square=\mathbf{0 , 2}$ & 434 & 3,09 & 0,9729 \\
\hline 5 & $\square=\mathbf{0 , 3}$ & 434 & 3,09 & 0,9729 \\
\hline 6 & $\square=\mathbf{0 , 4}$ & 434 & 3,09 & 0,9729 \\
\hline 7 & $\square=\mathbf{0 , 5}$ & 434 & 3,09 & 0,9729 \\
\hline 8 & $\square=\mathbf{0 , 6}$ & 434 & 3,09 & 0,9729 \\
\hline 9 & $\square=\mathbf{0 , 7}$ & 434 & 3,09 & 0,9729 \\
\hline 10 & $\square=\mathbf{0 , 8}$ & 430 & 2,14 & 0,9677 \\
\hline 11 & $\square=\mathbf{0 , 9}$ & 429 & 1,90 & 0,9656 \\
\hline
\end{tabular}

Fonte: Elaborado pelos autores. 
Tabela 4 - Quadro de Análise de Variância - Satisfação.

\begin{tabular}{cccccc}
\hline $\begin{array}{c}\text { Origem de } \\
\text { Variações }\end{array}$ & $\begin{array}{c}\text { Grau de } \\
\text { Liberdade } \\
\text { (GL) }\end{array}$ & $\begin{array}{c}\text { Soma de } \\
\text { Quadrados } \\
\text { (SQ) }\end{array}$ & $\begin{array}{c}\text { Quadrado } \\
\text { Médio } \\
\text { (QM) }\end{array}$ & $F$ & $\begin{array}{c}\text { F - crítico } \\
\square=0,05\end{array}$ \\
\hline $\begin{array}{c}\text { Entre } \\
\text { Grupos }\end{array}$ & 10 & 0,0262 & 0,0026 & 0,3555 & 1,8585 \\
$\begin{array}{c}\text { Dentro dos } \\
\text { Grupos }\end{array}$ & 341 & 2,5128 & 0,0074 & & \\
Total & 351 & 2,5390 & & & \\
\hline
\end{tabular}

Fonte: Elaborado pelos autores.

Através da Tabela 3, observa-se que, em todos os testes o modelo proposto apresenta melhores resultados em relação à designação manual. $\mathrm{O}$ aumento da satisfação global foi de em média $2,76 \%$ e o melhor índice médio foi de 0,9729 obtido em 7 dos 10 testes realizados.

Por outro lado, mediante a Tabela 4, nota-se que o valor da estatística F é inferior ao valor crítico, um indicativo que não há diferença significativa entre as amostras de índices de satisfação.

Relacionando os dados das Tabelas 1 e 3 nota-se que é possível obter índices de satisfação docente tão bons quanto o do modelo apresentado em Ferreira et al. (2011) $(\lambda=\mathbf{0})$, cujo objetivo é maximizar essa satisfação, com melhores índices de habilidade, como no caso dos testes com $\lambda=\mathbf{0}, \mathbf{2}, \boldsymbol{\lambda}=\mathbf{0}, \mathbf{3}, \boldsymbol{\lambda}=\mathbf{0}, \mathbf{4}, \boldsymbol{\lambda}=\mathbf{0}, \mathbf{5}, \boldsymbol{\lambda}=$ 0,6 e $\lambda=0,7$.

\subsection{CARGA HORÁRIA}

Para que o docente desenvolva um trabalho de qualidade é importante que o mesmo não possua uma carga horária em aulas muito elevada, de modo que este possa adicionar em sua agenda semanal um maior tempo para preparação de aulas, desenvolvimento de projetos de extensão e pesquisa, dentre outras atividades acadêmicas. Desse modo, considerou-se a carga horária como um dos fatores de análise de resultados. Na Tabela 5 são apresentadas as percentagens de professores que mantiveram ou diminuíram a sua carga horária em relação à designação manual.

Tabela 5 - Carga Horária.

\begin{tabular}{cc}
\hline Modelo & $\begin{array}{c}\text { Manteve ou } \\
\text { diminui (\%) }\end{array}$ \\
\hline$\square=0$ & 56,25 \\
$\square=0,1$ & 62,50 \\
$\square=0,2$ & 62,50 \\
$\square=0,3$ & 59,38 \\
$\square=0,4$ & 62,50 \\
$\square=0,5$ & 59,38 \\
$\square=0,6$ & 59,38
\end{tabular}


Tabela 5 - Carga Horária.

\begin{tabular}{cc} 
& (conclusão) \\
\hline Modelo & $\begin{array}{c}\text { Manteve ou } \\
\text { diminui (\%) }\end{array}$ \\
\hline$\square=0,7$ & 62,50 \\
$\square=0,8$ & 59,38 \\
$\square=0,9$ & 62,50
\end{tabular}

Fonte: Elaborado pelos autores.

Os melhores resultados foram apresentados pelos testes com $\lambda=\mathbf{0}, \mathbf{1}, \boldsymbol{\lambda}=$ $0,2, \lambda=0,4, \lambda=0,7, e \lambda=0,9$. Já o teste com $\lambda=0$ foi o que apresentou um maior número de docentes com carga horária aumentada.

Ao relacionar os dados das Tabelas 1, 3 e 5, verifica-se que não há um teste em que obteve um melhor resultado nos 3 fatores (habilidade, satisfação e carga horária), entretanto os testes $\operatorname{com} \lambda=\mathbf{0}, 2, \lambda=\mathbf{0 , 4}$ e $\lambda=\mathbf{0 , 7}$ foram os que apresentaram 0 melhor conjunto de resultados.

\section{CONSIDERAÇÕES FINAIS}

O problema de designação de encargos didáticos apesar de ser estudado desde a década de 60, ainda permanece atual e relevante, o que se deve às suas peculiaridades e complexidade, despertando interesse de estudiosos e pesquisadores.

A proposta apresentada no presente trabalho é a de um modelo de Programação Linear Inteira a este problema, em um contexto universitário, o qual permite-se a atribuição de disciplinas a professores habilitados em ministrá-las, prezando assim pela qualidade do processo de ensino-aprendizagem, de modo a respeitar as normas institucionais e otimizar a satisfação do corpo docente, considerando portanto, aspectos pedagógicos, organizacionais e pessoais, fundamentais para a modelagem e resolução deste tipo de problema.

Para o estudo de caso realizado, o modelo apresentado obteve bons resultados neste sentido. Ao comparar os resultados obtidos com a aplicação do modelo, com a designação manual, anteriormente realizada e também a outra formulação apresentada na literatura (a qual foi tomada como base para o desenvolvimento do trabalho), observa-se aumentos nos índices de habilidade e satisfação docente, além da manutenção ou diminuição da carga horária semanal de aulas dos professores, em todos os testes realizados, o que demonstra o potencial promissor do modelo desenvolvido.

Como perspectiva futura, sugere-se testes em um maior período de análise, expansão de departamentos atendidos; estudo de alternativas não manuais de calibração do modelo (parâmetro $\lambda$ ); comparativo com outros modelos, bem como, a utilização de uma interface gráfica ou até mesmo planilhas eletrônicas de forma a facilitar a utilização do modelo pelos gestores.

\section{REFERÊNCIAS}

ABDELLAHI, M.; ELEDUM, $H$. The university timetabling problem: modeling and 
solution using binary integer programming with penalty functions. International Journal of Applied Mathematics and Statistics, v. 56, n. 6, p. 164-178, 2017.

AKKOYUNLU, E. A. A linear algorithm for computing the optimum university timetable. The Computer Journal, v. 16, n. 4, p. 347-350, 1973.

AVELLA, P.; VASIL'EV, I. A computational study of a cutting plane algorithm for university course timetabling. Journal of Scheduling, v. 6, n. 8, p. 497-514, 2005.

BIRBAS, T.; DASKALAKI, S.; HOUSOS, E. Timetabling for Greek high schools. Journal of the Operational Research Society, v. 48, n. 12, p. 1191-1200, 1997.

BRESLAW, J. A. A linear programming solution to the faculty assignment problem. Socio-Economic Planning Sciences, v. 10, n. 6, p. 227-230, 1976.

CARVALHO R. Abordagem heurística para o problema de programação de horários de cursos. 2011. Dissertação (Mestrado em Engenharia Elétrica). Universidade Federal de Minas Gerais, Belo Horizonte, 2011.

CSIMA, J.; GOTLIEB, C. C. Tests on a computer method for constructing school timetables. Communications of the ACM, v. 7, n. 3, p. p 160-163, Mar. 1964. phttps://doi.org/10.1145/363958.363986.

DOMENECH, B.; LUSA, A. A MILP model for the teacher assignment problem considering teachers' preferences. European Journal of Operational Research, $v$. 249, n. 3, p. 1153-1160, 2015.

EVEN, S.; ITAI, A.; SHAMIR A. On the complexity of time table and multi-commodity flow problems problems. In: ANNUAL SYMPOSIUM ON FOUNDATIONS OF COMPUTER SCIENCE, $16^{\text {TH }}$., 1975. Proceedings [...]. [s.I.]: IEEE, 1975, p. 184193. doi: $10.1109 /$ SFCS.1975.21.

FERREIRA, P. S. et al. Aplicação de programação inteira na distribuição de encargos didáticos em instituições de ensino. Trends in Applied and Computational Mathematics, v. 12, n. 2, p. 135-144, 2011. Doi: 10.5540/tema.2011.012.02.0135

GOTLIEB, C.C. The construction of class-teacher time-tables. In: PROCEEDINGS OF INTERNATIONAL FEDERATION FOR INFORMATION PROCESSING (IFIP), 62., Amsterdam. Anais [...]. Munich: North Holland Publishing Company, 1963, p.73-77.

HEITMANN, H.; BRÜGGEMANN, W. Preference-based assignment of university students to multiple teaching groups. OR Spectrum, v. 36, n. 3, p. 607-629, 2013.

LAWRIE, N. L. An integer linear programming model of a school timetabling problem. The Computer Journal, v. 12, n. 4, p. 307-316, 1969.

PILLAY, N. A survey of school timetabling research. Annals of Operations Research, v. 218, n. 1, 2013. p. 261-293.

UTFPR. Diretrizes para a Gestão das Atividades de Ensino, Pesquisa e 
Extensão da UTFPR. Curitiba: UTFPR, 2007.

VIEIRA, S. Análise de variância: ANOVA. São Paulo: Atlas, 2006.

WELSH, D. J. A.; POWELL, M. B. An upper bound for the chromatic number of a graph and its application to timetabling problems, The Computer Journal, v.10, n.1, p. 85-86, 1967. 